\title{
Burden of Neisseria meningitidis infections in China: a systematic review and meta-analysis
}

\author{
Yaowen Zhang ${ }^{1}$, Dong $\mathrm{Wei}^{1}$, Xinzhen \\ $\mathrm{Guo}^{2}$, Mai Han², Lichao Yuan², Moe H \\ Kyaw $^{3}$ \\ ${ }^{1}$ Infection Management and Disease Prevention \\ Department, China-Japan Friendship Hospital, \\ Hepingli, Beijing, China \\ ${ }^{2}$ Department of Infectious Diseases, China-Japan \\ Friendship Hospital, Hepingli, Beijing, China \\ ${ }^{3}$ Sanofi Pasteur, Discovery Drive, Swiftwater, PA, USA
}

Background Neisseria meningitidis is a leading cause of bacterial meningitis and septicemia in children and young adults worldwide. The disease burden associated with $N$. meningitidis infections has not been systematically assessed in China. Therefore, we undertook this study to determine the burden of meningococcal disease in China.

Method We performed a systematic review and meta-analysis of articles on N. meningitidis incidence, carriage, seroprevalence and mortality rates in China by searching the Chinese BioMedical Database (CBM), China National Knowledge Infrastructure (CNKI), Wanfang database and PubMed for publications from January 2005 to Aug 2015.

Results In total, 50 articles were included in our analysis. The overall incidence of meningococcal disease and associated mortality were estimated to be 1.84 (95\% confidence interval (CI) 0.91-3.37) per 100000 persons per year and 0.33 (95\% CI 0.12-0.86) per 100000 persons per year, respectively. N. meningitidis carriage rate among the healthy population was estimated to be $2.7 \%$ (95\% CI 2.0-3.5\%). Prevalence of antibodies against $N$. meningitidis serogroup A and C were estimated to be $77.3 \%$ (95\% CI $72.4 \%-81.6 \%$ ) and $33.5 \%$ (95\% CI 27.0\%-40.8\%), respectively. No studies were found for serogroup specific disease burden.

Conclusions The overall incidence of meningococcal disease in China is low. The lower seroprevalence of serogroup $C$ within the population suggests that it may pose a greater risk for meningococcal disease outbreak than serogroup A. The lack of data on serogroup disease burden by age groups suggests the implementation of laboratory based meningococcal surveillance systems are urgently needed in China.

Neisseria meningitidis, a Gram-negative diplococcus, asymptomatically colonizes the upper respiratory tract of approximately $10 \%$ of healthy humans, and is a leading cause of bacterial meningitis and septicemia in children and young adults worldwide [1,2]. It is transmitted through direct contact with respiratory secretions or aerosol droplets released by coughing/sneezing from patients with meningococcal disease or asymptomatic carriers. Humans are the only host [3]. Most cases of meningococcal disease are caused by serogroups A, B, C, Y and W [4]. Meningitis caused by $N$. meningitidis continues to be a serious threat to global health, account- 
ing for 1.2 million cases and 135000 deaths worldwide each year [5], despite the existence of effective vaccines [6].

Serogroup A has historically been the dominant serogroup in China accounting for over 95\% of meningococcal disease cases from the 1960s to 1980s, with annual disease incidence rates up to 400 cases per 100000 population in some regions $[7,8]$. Following the introduction of a serogroup A polysaccharide meningococcal vaccine into the national immunization program in 1982, the incidence rates in the subsequent two decades were reduced and remained low and relatively stable ranging $0.2-1$ cases per $100000[9,10]$. However, during 2003-2004, serogroup C (type ST-4821) meningococcal disease outbreaks emerged in the Anhui province [11]. This new strain appeared more invasive, causing serious complications more frequently and was associated with a higher case-fatality rate than serogroup A [12]. Serogroup C meningococcal disease quickly became endemic in the Anhui Province, with ST-4821 the dominant lineage. This lineage rapidly spread nationwide causing several meningococcal disease outbreaks in 2004-2005 [12,13]. In response to these outbreaks, meningococcal group $\mathrm{A}$ and $\mathrm{C}$ polysaccharide vaccines were subsequently used for routine immunization. Nonetheless, serogroup C continues to be isolated every year throughout China with ST-4821 dominant [14].

Understanding changes in epidemiology of meningococcal disease after use of meningococcal A/C polysaccharide vaccines and $N$. meningitidis carriage rates and seroprevalence can help predict the potential public health impact of routine vaccination. To date, the disease burden associated with $N$. meningitidis infections has not been systematically assessed in China. We therefore conducted this systematic review and meta-analysis to evaluate the incidence of meningococcal disease and associated mortality, as well as carriage rates and prevalence of antibodies against $N$. meningitidis in China.

\section{METHODS}

\section{Search strategy}

We undertook a systematic search across the following electronic databases: Chinese BioMedical Database (CBM), China National Knowledge Infrastructure (CNKI), Wanfang database and PubMed. The specific details of the search strategies undertaken across these databases are presented in Appendix S1 in Online Supplementary Document. In brief, the following search terms were used to search the databases: "meningococcus", "meningococcal", "meningococcic" and "meningitidis". The search in PubMed included 'China' as a search term. We restricted our search to articles published in Chinese "core journals", as listed by the Peking University (2014 edition) and evaluated according to predefined criteria; journals considered to be of low quality are excluded from the list [15]. We focused our search on recent data, published from January 2005 to August 2015.

\section{Inclusion and exclusion criteria}

Studies were selected for inclusion based upon the following criteria: 1) included humans; 2) reported at least one outcome relevant to our study objectives; 3) published in Chinese or English language. We excluded case reports and other systematic reviews or meta-analyses. Where multiple studies on the same cohort were identified, the latest publication or that with the most complete data was included in our meta-analysis.

\section{Literature screening and data extraction}

Two groups of reviewers screened the titles, keywords and abstracts of the citations identified (DW and XG independently reviewed records 1-1850; MH and LY independently reviewed records 1851-3703) and excluded those that clearly did not meet the inclusion criteria. YZ screened the citations identified in a similar manner. The full texts of all selected publications were assessed for relevance. Any disagreement or uncertainty between the reviewers about the eligibility of a study was resolved by $\mathrm{YZ}$, and in the case of persistent disagreement, the full text of the article was examined. The reference lists of articles identified for inclusion were inspected for other appropriate articles not identified by the electronic search.

The reviewers independently extracted and entered data from each included study into a database. The following data were extracted from the studies where available: authors; year of publication; study design; study period; location of the study; number of meningococcal disease cases or reported incidence (crude and age-specific); number of deaths or reported death rates; $N$. meningitidis carriage rates; and prevalence of antibodies against $N$. meningitidis and corresponding serogroup. The data extracted were checked for inconsistencies between the reviewers and resolved by a fifth author. We did not attempt to contact the authors of the studies identified for missing information or resolve ambiguities.

\section{Quality assessment}

The analysis included studies with different outcomes. Therefore, no pre-existing scale is directly suit able for the quality assessment. The quality of each included study was assessed by $\mathrm{YZ}$ and DW using predefined criteria as previously described [16]. In brief, the quality of the studies was based on the clarity of information provided on the following 5 items scored on a three-point scale (from 0 - poorest 
to 2 - best quality): population and representativeness; diagnostic criteria; specimen collection methods; pathogen or antibody detection methods; and statistical methods. The scoring is defined as 2 points for detailed reporting, 1 point for non-detailed reporting and zero point for no reporting of the selected criteria for the assessment. The score for each item was then added to give a composite score for the study, with a highest total score of 10 . Studies with total scores $\geq 8$ were regarded as "good" quality.

\section{Statistical analysis}

All meta-analyses were performed using the MetaAnalyst (Beta 3.13; http://tuftscaes.org/meta_analyst) software package. Since we were expecting considerable heterogeneity across the included studies, we used the random-effects model of the Der-Simonian Laird method. Publication bias was investigated via Stata 12.0 (StataCorp LP, Texas, USA) using Egger's test.

\section{RESULTS}

\section{Studies included}

The electronic search identified 3703 citations (Figure 1). After removal of non-Chinese core journals and duplicates, and screening titles and abstracts, 90 studies were judged as potentially relevant with the full text retrieved to assess their eligibility for inclusion. Overall, 50 studies [17-66] met the eligibility criteria and were thus included in our analysis. These studies reported data collected from 1991 to 2013. The quality evaluation score for these studies ranged from 5 to 10 points, with a mean \pm standard deviation of $8.1 \pm 1.2$. There were $36(72 \%)$ studies with a score of $\geq 8$. A summary of the characteristics of included studies associated with their respective quality assessment is presented in Appendix S2 in Online Supplementary Document. Studies included the analysis are listed in Appendix S3 in Online Supplementary Document.

\section{Meningococcal disease incidence and associated mortality rate}

Eleven studies [21,24,25,29,44,46,48,57-60] conducted in 7 provinces provided data on the meningococcal disease incidence and associated mortality rate. The annual meningococcal disease incidence and mortality rate for a representative period between 2000 and 2010 is summarized in Table1. The highest incidence rates occurred in the years 2006, 2007 and 2010; the incidence rates reported in these years were $>2.0$ per 100000 persons per year. The corresponding highest mortality rates occurred in the years

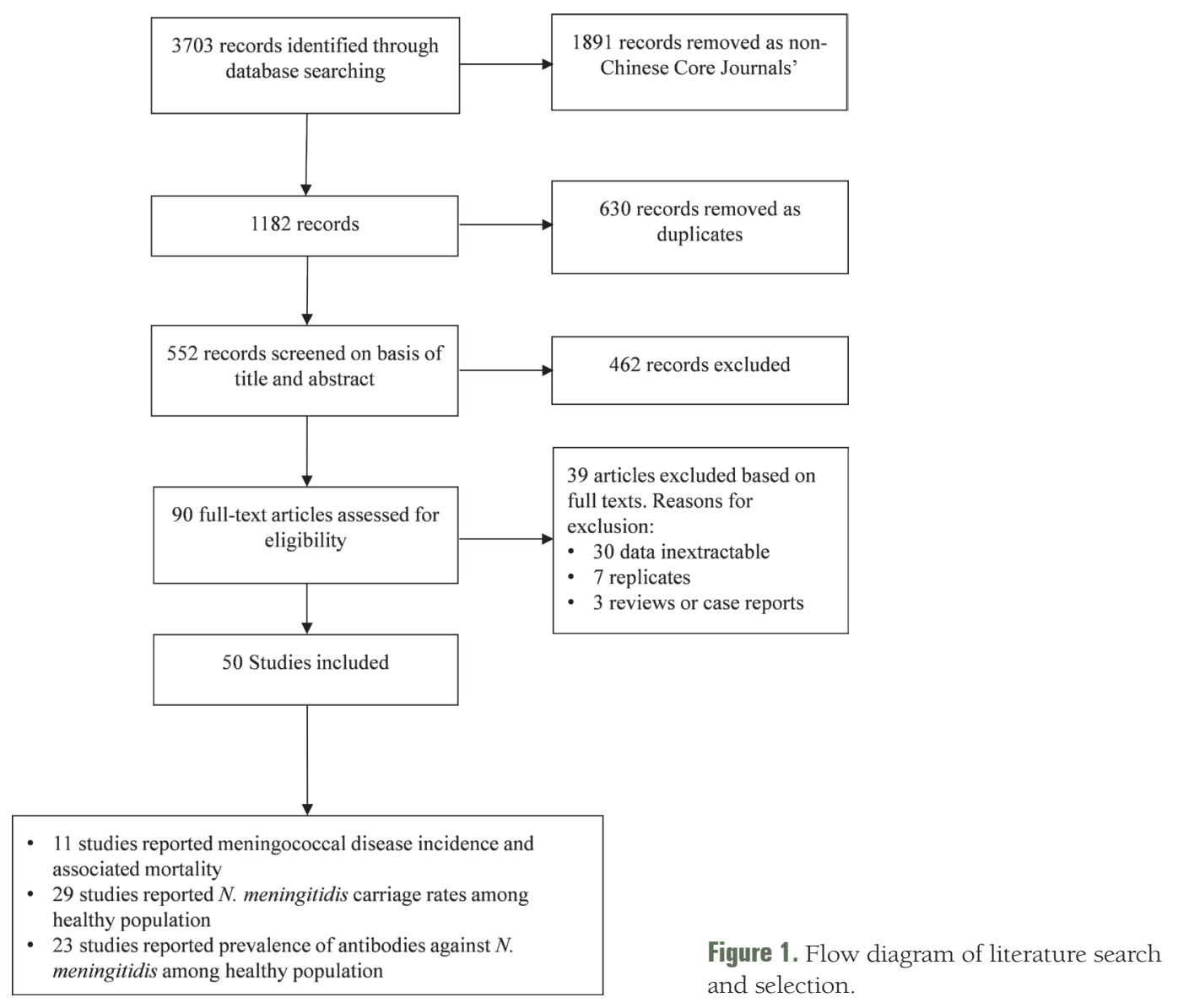


Table 1. Meta-analysis of annual meningococcal disease incidence and associated mortality rate from 2000 to 2010 (per 100000 persons per year)

\begin{tabular}{ccccc} 
YeAR & \multicolumn{2}{c}{ IneIDENGE } & \multicolumn{3}{c}{ MoRTALIT } \\
& $\begin{array}{c}\text { Number } \\
\text { of studies }\end{array}$ & Rate & $\begin{array}{c}\text { Number } \\
\text { of studies }\end{array}$ & Rate \\
2000 & 5 & $0.71(0.18-2.44)$ & 3 & $0.37(0.02-3.79)$ \\
\hline 2001 & 5 & $0.66(0.17-2.28)$ & 3 & $0.01(0.00-9.96)$ \\
\hline 2002 & 5 & $0.91(0.23-3.00)$ & 3 & $0.44(0.06-2.52)$ \\
\hline 2003 & 6 & $1.23(0.31-3.85)$ & 3 & $0.44(0.66-2.52)$ \\
\hline 2004 & 7 & $1.09(0.19-4.33)$ & 3 & $0.02(0.00-9.18)$ \\
\hline 2005 & 10 & $1.07(0.26-3.53)$ & 4 & $0.02(0.00-7.82)$ \\
\hline 2006 & 8 & $2.18(0.69-5.13)$ & 4 & $0.02(0.00-7.68)$ \\
\hline 2007 & 8 & $2.30(0.76-5.21)$ & 4 & $0.01(0.00-8.91)$ \\
\hline 2008 & 5 & $1.41(0.17-6.14)$ & 3 & $0.01(0.00-9.97)$ \\
\hline 2009 & 5 & $1.88(0.53-4.89)$ & 3 & $0.40(0.03-4.01)$ \\
\hline 2010 & 3 & $2.17(0.06-5.42)$ & 2 & $0.44(0.03-4.35)$ \\
\hline Total & 11 & $1.84(0.91-3.37)$ & 5 & $0.33(0.12-0.86)$ \\
\hline
\end{tabular}

2002, 2003 and 2010; the mortality rate reported in these years was 0.44 per 100000 persons per year. The average incidence of meningococcal disease and associated mortality during the study period assessed were estimated to be 1.84 (95\% confidence interval (CI) 0.91-3.37) per 100000 and 0.33 (95\% CI 0.12-0.86) per 100000 , respectively.

\section{$N$. meningitidis carriage rate among the healthy population}

Twenty-nine studies [17-19, 22, 24, 26-28, 30-32, 34, 35, $37,39-44,47,49,51,52,56,57,62,64,65]$ conducted in 14 provinces reported $N$. meningitidis carriage rates among the healthy population. The analysis for N. meningitidis carriage rate included 1248 positive cases identified from 45462 throat swabs from healthy people between 2000 and 2013 , representing an overall carriage rate of $2.7 \%$ (95\% CI $2.0 \%-3.5 \%)$.

\section{Prevalence of antibodies against $\mathbf{N}$. meningitidis among the healthy population}

Twenty-three studies [20, 22, 23, 28, 30, 33, 34, 36, 38, 39, $42,43,45,50,52-55,57,61,63,65,66]$ conducted in 11 provinces reported the prevalence of antibodies against $N$. meningitidis among the healthy population. The age-specific prevalence of antibodies against N. meningitidis is summarized in Table 2. Serogroup A specific N. meningitidis were generally highest in those aged 5-24 years and for serogroup $\mathrm{C}$ it was highest in those aged 25 years or older. The overall prevalence of antibodies against $\mathrm{N}$. meningitidis serogroup A and $\mathrm{C}$ was $77.3 \%$ (95\% CI $72.4 \%-81.6 \%$ ) and $33.5 \%$ (95\% CI $27.0 \%-40.8 \%$ ), respectively, for the period 2001-2012.

\section{Sensitivity analysis and publication bias}

Sensitivity analyses undertaken to include only 'good' quality studies did not significantly alter the outcomes. Overall
Table 2. Meta-analysis of age-specific prevalence of antibodies against $N$. meningitidis among the healthy population

\begin{tabular}{|c|c|c|c|c|}
\hline $\begin{array}{l}\text { AgE-GROUP } \\
\text { (YeARS) }\end{array}$ & $\begin{array}{l}\text { NUMBER of } \\
\text { STUDIES }\end{array}$ & $\begin{array}{l}\text { Antiboov- } \\
\text { Positilve }\end{array}$ & $\begin{array}{l}\text { TOTAL NUMBER } \\
\text { OF PARTICIPANIS }\end{array}$ & $\begin{array}{l}\text { PositIVE RATE (\%) } \\
\text { (95\% CI) }\end{array}$ \\
\hline \multicolumn{5}{|c|}{ Meningococcal serogroup A: } \\
\hline $0-4$ & 22 & 3101 & 4701 & $70.9(63.4-77.4)$ \\
\hline $5-9$ & 15 & 1367 & 1722 & $81.4(75.5-86.2)$ \\
\hline $10-14$ & 15 & 1515 & 1912 & $78.2(72.4-83.1)$ \\
\hline $15-24$ & 15 & 1623 & 2071 & $78.2(71.7-83.6)$ \\
\hline $25-34$ & 14 & 1101 & 1488 & $75.0(66.1-82.2)$ \\
\hline $35-44$ & 14 & 1058 & 1357 & $76.3(65.3-84.7)$ \\
\hline $45-$ & 14 & 1038 & 1424 & $72.5(61.6-91.2)$ \\
\hline Total & 22 & 10803 & 14676 & $77.3(72.4-81.6)$ \\
\hline \multicolumn{5}{|c|}{ Meningococcal serogroup C: } \\
\hline $0-4$ & 22 & 1658 & 4395 & $23.6(16.0-33.5)$ \\
\hline $5-9$ & 14 & 610 & 1367 & $34.9(23.5-48.4)$ \\
\hline $10-14$ & 14 & 533 & 1347 & $24.1(14.7-36.9)$ \\
\hline $15-24$ & 14 & 606 & 1539 & $32.0(24.4-40.7)$ \\
\hline $25-34$ & 13 & 616 & 1347 & $39.9(31.9-48.5)$ \\
\hline $35-44$ & 13 & 656 & 1210 & $42.6(32.2-53.7)$ \\
\hline $45-$ & 13 & 703 & 1283 & $46.7(37.0-56.6)$ \\
\hline Total & 22 & 5382 & 12488 & $33.5(27.0-40.8)$ \\
\hline
\end{tabular}

$\mathrm{CI}$ - confidence interval

N. meningitidis carriage rate among the healthy population reported in 22 good quality studies was 3.2\% (95\% CI, $2.4 \%-4.2 \%)$ and the prevalence of antibodies against $N$. meningitidis serogroup $\mathrm{A}$ and $\mathrm{C}$ among the healthy population reported in 20 good quality studies were $75.9 \%$ (95\%CI $70.5 \%-80.6 \%)$ and $34.3 \%(27.5 \%-42.0 \%)$, respectively. The Egger's test did not reveal any significant publication bias $(-0.51,95 \% \mathrm{CI}-4.72$ to $2.87, P=0.616)$ for $N$. meningitidis carriage rate among the healthy population. As the majority of studies were captured in the analysis of carriage data, we did not consider further sensitivity analysis of the other data to be necessary.

\section{Discussion}

Our study is the first to systematically review, collate and analyze available published studies on the disease burden of N. meningitidis infections in China using robust metaanalytical methods. We found that the incidence of meningococcal disease and associated mortality are low ranging $0.66-2.30$ per 100000 persons per year and $0.01-0.44$ per 100000 , respectively. The incidence of meningococcal disease from our study is consistent with that reported in developed countries, typically $<2$ per 100000 , but lower than that reported in developing countries (typically $>10$ per 100 000), particularly in Africa [67].

In China, meningococcal disease historically occurred in a cyclical pattern at intervals of 8-10 years, with nationwide epidemics in 1959, 1967, 1977, and $1984[7,68]$. The spring of 1967 had the highest incidence of meningococcal disease with reported rates of 403 per 100000 , corresponding to more than 3.04 million cases. The associated 
mortality rate in that year was $5.5 \%$ corresponding to more than 160000 deaths [69]. The epidemic in 1977 had an incidence of 59.7 per 100000 and a $4.0 \%$ fatality rate. These deadly cyclic epidemics and seasonal patterns clearly highlight the unpredictability of outbreaks of meningococcal disease despite our observed low incidence rates in the study period.

In our analysis, the prevalence of antibodies against N. meningitidis serogroup C was $33.5 \%$ and tended to increase with age, whereas the prevalence of serogroup A antibodies was highest in the 5-9 year age group and decreased with age. The lower seroprevalence of serogroup $C$ within the population suggests that it may pose a greater risk for meningococcal disease outbreak than serogroup A, particularly in the youngest age group (less than 5 years) who have the lowest seroprevalence. Despite over 90\% uptake for meningococcal serogroup $\mathrm{A} / \mathrm{C}$ polysaccharide vaccines, the observation of low seroprevalence against serogroup A and $C$ in children less than 5 years old suggests that the implementation of conjugate meningococcal vaccine is necessary particularly for those less than 2 years old to whom the polysaccharide meningococcal vaccines have limited benefits and protection.

We estimated the nasopharyngeal $N$. meningitidis carriage to be $2.7 \%$, which appear lower than the generally quoted overall rate of $10 \%$ [70], and the average 3.5-35\% reported in studies conducted in Africa [71], but at least consistent with that reported in Mexico (1.6\%) [72]. The low carriage rates found in our study and those reported in Mexico are consistent with the low disease incidence rate reported in these two countries. Currently the burden of meningococcal disease in Mexico is low, with total national cases as low as two per year $[73,74]$. The prevalence of $N$. meningitidis carriage in healthy children and adolescents aged 10-19 years in Chile was reported to be 6.5\% [75], and slightly lower in university students ages 18-24 years (4\%) [76]. The corresponding incidence of meningococcal disease in Chile was also low, varying from $0.33-0.59$ per 100000 in the six years up to 2012 [77]. A European meta-analysis including 143114 individuals found that the carriage rate increased from $4.5 \%$ in infants to $24 \%$ in 19 -year olds and decreased to $8 \%$ in 50-year old adults [78]. Data on age-specific carriage rates in China are currently lacking. Since information on carriage rates is important for understanding the epidemiology and transmission of meningococcus and developing vaccination strategies, studies on age-specific carriage rates are recommended in China.
Our study has a number of limitations that should be considered. The meta-analysis was based on observational studies and as such is constrained by the inherent heterogeneity in such studies (for example, differences in sampling techniques, laboratory methods, age groups assessed, period of study/seasonality effects) and underlying confounding factors. In addition, we combined data from different regions and time periods. To account for this, we adopted a random-effects model to pool all results, leading to a wider $95 \% \mathrm{CI}$ that provided a more conservative estimate of the overall results. There were few studies found for certain geographic regions of west China, such as Gansu, Xinjiang and Tibet, which may also contribute to low precision estimates in those areas and overall. We did not search the gray literature; therefore, data that were not published in the 4 selected search databases may have been missed. Nevertheless, our study is likely to capture all important Chinese data on meningococcal disease since we included all available core journals in our study. Other shortcoming are, we only considered studies published in English and Chinese and did not contacted to authors for missing information or resolve ambiguities. However, it is unlikely there would be any significant literature in other languages. Since most important study data were available to obtain during data extraction, we feel that clarifications with the authors are not necessary and do not expect to have any impact on the study results. In addition, there were no studies identified that reported disease burden by serogroup, which limits our understanding of seroepidemiology of meningococcal disease and for developing recommendations for the selection of meningococcal vaccines in different age groups. Nevertheless, almost all meningococcal disease was caused by serogroup A and C based on the limited available data [11,79]. Therefore, the use of meningococcal conjugate vaccines including serogroups $\mathrm{A} / \mathrm{C} / \mathrm{W} / \mathrm{Y}$ can further reduce the burden of meningococcal disease and prevent the occurrence of large outbreaks in China.

In conclusion, although the overall incidence of meningococcal disease in China is low, the lower seroprevalence of serogroup $C$ within the population suggest that it may pose a greater risk for meningococcal disease outbreak than serogroup A, especially for children aged less than 5 years. The lack of data on serogroup-specific disease burden by age group suggests that the implementation of laboratorybased meningococcal surveillance systems is urgently needed in China. 
Acknowledgments: Editorial assistance with the preparation of the manuscript was provided by Richard Glover, in Science Communications, Springer Healthcare. Funding for this assistance was provided by Sanofi Pasteur. We thank Walter Sella, MD of Sanofi Pasteur for assistance with the publication process of this manuscript.

Funding: This study was sponsored by Sanofi Pasteur.

Authors' contributions: Study design (MHK, YZ); data collection (DW, XG, MH, LY); data analysis and interpretation (YZ, DW, XG, MH, MHK); development of initial draft of manuscript (YZ, MHK), critical revisions for intellectual content of manuscript (YZ, DW, XG, MH, LY, MHK); study supervision (YZ, MHK). All authors approved the final manuscript for submission, and are responsible for the veracity and completeness of the data reported.

Competing interests: All authors have completed the Unified Competing Interest form at www.icmje. org/coi_disclosure.pdf (available on request from the corresponding author) and declare no conflict of interest. MHK is an employee of Sanofi Pasteur.

1 Rosenstein NE, Perkins BA, Stephens DS, Popovic T, Hughes JM. Meningococcal disease. N Engl J Med. 2001;344:1378-88. Medline:11333996 doi:10.1056/NEJM200105033441807

2 DeVoe IW. The meningococcus and mechanisms of pathogenicity. Microbiol Rev. 1982;46:162-90. Medline:6126800

3 Stephens DS, Zimmer SM. Pathogenesis, therapy, and prevention of meningococcal sepsis. Curr Infect Dis Rep. 2002;4:377-86. Medline:12228024 doi:10.1007/s11908-002-0004-4

4 Harrison LH, Trotter CL, Ramsay ME. Global epidemiology of meningococcal disease. Vaccine. 2009;27 Suppl 2:B51-63. Medline:19477562 doi:10.1016/j.vaccine.2009.04.063

5 World Health Organization. Epidemics of meningococcal disease. African meningitis belt, 2001. Wkly Epidemiol Rec. 2001;76:282-8. Medline:11577498

6 World Health Organization. Meningococcal vaccines: WHO position paper, November 2011. Wkly Epidemiol Rec. 2011;86:521-39. Medline:22128384

$7 \mathrm{Hu}$ X. [Study on periodically prevalent feature for epidemic cerebrospinal meningitis in China]. [Article in Chinese]. J Chin Epidemiol. 1991;12:136-9. Medline:1907529

$8 \mathrm{Hu}$ Z. [Analysis of group A meningococcal surveillance and cloning of China in 1980]. [Article in Chinese]. Chin J Publ Health. 1994;13:90-2.

9 Jin YH, Wang B. [The epidemiological trend of meningococcal disease in Hefei, 1990-1999.] [Article in Chinese]. Chin J Dis Control Prevent. 2001;5:157-8.

10 Yang JF, Li JH. Y.X. L. [Prevention and control of epidemic meningococcal disease]. [Article in Chinese]. Chin J Vaccine Immun. 2006;12:61-3.

11 Shao Z, Li W, Ren J, Liang X, Xu L, Diao B, et al. Identification of a new Neisseria meningitidis serogroup C clone from Anhui province, China. Lancet. 2006;367:419-23. Medline:16458767 doi:10.1016/S01406736(06)68141-5

$12 \mathrm{Xu} \mathrm{XH}$, Ye Y, Hu LF, Jin YH, Jiang QQ, Li JB. Emergence of serogroup C meningococcal disease associated with a high mortality rate in Hefei, China. BMC Infect Dis. 2012;12:205. Medline:22943188 doi:10.1186/1471-2334$12-205$

13 Zhou H, Gao Y, Xu L, Li M, Li Q, Li Y, et al. Distribution of serogroups and sequence types in disease-associated and carrier strains of Neisseria meningitidis isolated in China between 2003 and 2008. Epidemiol Infect. 2012;140:1296-303. Medline:21929839 doi:10.1017/S0950268811001865

14 Shan X, Zhang J, Zhou H, Zhu B, Xu L, Shao Z, et al. Genetic diversity of Neisseria meningitidis serogroup C ST-4821 in China based on multiple-locus variable number tandem repeat analysis. PLoS One. 2014;9:e111866. Medline:25375168 doi:10.1371/journal.pone.0111866

15 The Library of Perking University. Chinese core journals, 2014 edition. Available: http://www.zzqklm.com/w/ qz/15567.html. Accessed: 2 November 2015.

16 Zhang Y, Yuan L, Zhang X, Zheng M, Kyaw MH. Burden of respiratory syncytial virus infections in China: Systematic review and meta-analysis. J Glob Health. 2015;5:020417. Medline:26682049 doi:10.7189/ jogh.05.020417

17 Zhou ZR, Liang JL, Long DL, Li Q, Zhao XZ, Liu HC. Carrying Rate of Neissria Meningitides in a healthy population in the Mianzhu Post-earthquake residential area. J Sichuan Univ. 2009;40:716-8. Med Sci Edi. Medline: 19764581

18 Zhang XC, Zhu KR, Huang RN, Long HY. Evaluation of a rapid real-time PCR assay for detection the bacteria carrier level of Neisseria Meningitides in health population in Chengdu city. Mod Prev Med. 2009;36:1710-2.

19 Li Q, Wang XL, Zhang YM, Yang H. Zhao XZ. A survey of taking Neissria Meningitides in victims of Wenchuan earthquake in Mianzhu. Chinese Journal of Health Laboratory Technology. 2008;18:2723-4. 
20 Zhang XQ, Deng ZA, Zhou XZ, Li XQ, Li CH, Lin YW. Investigation on the immune level of the Menitis bacterium types $\mathrm{A}$ and $\mathrm{C}$ among the normal people in Fanyu, Guangzhou. Chinese Journal of Health Laboratory Technology. 2005;15:1360-01.

21 Dai B, Ni JD, Jin YH, Bai RJ, Zheng N, Ye DQ. Epidemiological characteristics of meningococcal meningitis in Hefei city during 2005 and 2007. Chin J Publ Health. 2009;25:79-80.

22 Zheng CZ, Shao RB, Bi C, Xun YB, Ma YF. Surveillance for the carrier of Neisseria Meningitis group A and C and antibody level among healthy people in Yancheng city. Mod Prev Med. 2012;39:1541-52.

23 Zheng CZ, Wang B, Shao RB, Bi C, Chen CB, Cai JP, et al. Investigation on the immune level of the menitis bacterium types A and C among healthy people. Mod Prev Med. 2009;36:3949-52.

24 Feng L, Xiong P, Li MS, Song LZ, Zhang Y, Liu GF, et al. Analysis on the epidemiological characteristics and the etiological surveillance for epidemic cerebrospinal Meningitis in Shandong province from 2006 to 2013. Chin J Epidemiol. 2014;35:1407-8.

25 Zhang TG, He X, Chen LJ, He JG, Yang J, Sun MP, et al. Surveillance on pathogens of Meningococcal Meningitis in Beijing, 2005. Chin J Epidemiol. 2006;27:396-8. Medline:16981333

26 Zhou ZR, Zhao XZ, Long DL, Deng Q, Li Q, Liu HC. Survey of germ-carrying rate of Neissria Meningitides in one prefab community in disaster area. Mod Prev Med. 2010;37:3338-40.

27 Long DL, Yao P, Gao WF, Wang ZX, Gao RR, Zhou ZR, et al. Survey on prevalence of Neisseria Meningitides among students in primary and middle schools in SiChuan distress area. Mod Prev Med. 2011;38:476-81.

28 Lin Z, Liu YY, Ding C, Chang XL, Liu TY, Li JC, et al. Survey of Neissria mealingitidis Carrier in healthy people in Tongliao. Chin J Publ Health. 2006;22:1500.

29 Long J, Zhao H, Li Q, Luo XJ, Xiao BZ, Yi J. Analysis of surveillance on epidemic cerebrospinal Meningitis from 2000 to 2005 in ChongQing city. Mod Prev Med. 2007;34:1652-5.

30 Luo XC, Luo LM, Gong Y, Luo HH, Cai SW, Zhao Q. The prevalence characteristics of epidemic cerebrospinal Meningitis in Sanming city in 2005. Chinese Journal of Health Laboratory Technology. 2006;16:451-2.

31 Luo SH, Zhang LP, Huang SZ, Li JQ. The prevalence characteristics of epidemic cerebrospinal Meningitis from 2000 to 2005 in Dongguan city. Chinese Journal of Health Laboratory Technology. 2006;16:701-2.

32 Luan L, Zhan YH, Zhang MH, Bi C, Zhu YH, Zhang J, et al. Epidemiological analysis of the meningococcal disease in Suzhou city during 1992-2012. Mod Prev Med. 2014;41:2113-8.

33 Jiang X, Qian ZY, Zhen SU, Wang YT, Jia ZY, Sun YQ, et al. Analysis of the antibody level of epidemic cerebrospinal meningitis among healthy population in HeBei province in 2008. Mod Prev Med. 2011;38:3415-57.

34 Liu MZ, Yang HK, Liao GD, Huang ZS, Xu L, Deng XL, et al. Investigation on antibody level against epidemic cerebrospinal meningitis and carriage rate of Neisseria Meningitidis in healthy population in Guangdong province. Chinese Journal of Health Laboratory Technology. 2007;17:325-38.

35 Tang XL. Investigation on germ-carrying situation on Neisseria Meningitidis of healthy populations in Qinghai province in 2007. Mod Prev Med. 2010;37:555-6.

36 Yang HK, Zhang LP, Liu MZ. Surveillance analyses on epidemic cerebrospinal Meninqitis of Dongguan in 2005. Chinese Journal of Health Laboratory Technology. 2006;16:76-77.

37 Yang Y, Liang JL, Ju CY, Su HW, Chen ZQ, Gan ZL, et al. Investigation on the germ-carrying status of epidemic Meningitis among health rural students in primary and middle school of Luzhou. Mod Prev Med. 2008;35:2347-9.

38 Jiang WP, Wang YJ. Analysis on antibody level against epidemic cerebrospinal Meningitis serogroup A and C in Danyang. Chin J Dis Control Prev. 2005;9:348-77.

39 Wang J, Yan LQ. Surveillance analyses on epidemic cerebrospinal Meninqitis of Zhongwei city of Ningxia in 2006. Chinese Journal of Health Laboratory Technology. 2007;17:495-6.

40 Fan YS, Zhang LS, Wang JH. Survey of N. mealingitidis Carrier in healthy people in Tangshan. Chinese Journal of Health Laboratory Technology. 2008;18:690-1.

41 Wu XH, Lin J, Quan Y, Fang JS, Liang DB, Qin WW, et al. Research on bacteria carrying, antibody level and control strategy for epidemic cerebrospinal Meningitis in Guangxi. Chin J Sch Health. 2010;31:337-9.

42 Liu DQ, Wang JJ, Wang BB, Chen X, Luo XW, Yan KL, et al. The prevalence characteristics of epidemic cerebrospinal Meningitis of serogroup C in Anhui Province. Chin J Dis Control Prev. 2010;14:240-4.

$43 \mathrm{Ju}$ CY. Genetic relationships among carried meningococci and the immunity of healthy people in Shenzhen, China [dissertation]. Sichuan: Sichuan University; 2007.

$44 \mathrm{Ni}$ JD. Study on the epidemiological characteristics of Meningococcal disease in Anhui province [doctoral thesis]. Anhiu: Anhui Medical University; 2008.

45 Xu BX, Yao PP, Wang FS. Analysis and survey of antibodies against neisseria meningitides in healthy population in Zhejiang province. Chinese Journal of Health Laboratory Technology. 2013;23:195-9.

$46 \mathrm{Xu} \mathrm{XH.} \mathrm{Epidemiology} \mathrm{and} \mathrm{molecular} \mathrm{epidemiology} \mathrm{of} \mathrm{Meningococcal} \mathrm{Meningitis} \mathrm{during} \mathrm{2000-2010} \mathrm{in} \mathrm{Hefei}$ city of Anhui province [doctoral thesis]. Anhui: Anhui Medical University; 2014.

47 Zhang JM, Jin WE, Ye CH. Situation of carrier rate of Neisseria Meningtitides in school health population in Quzhou city during 2003-2008. Chinese Journal of Health Laboratory Technology. 2013;19:1647-69.

48 Dai B. Study on the epidemiological characteristics of Meningococcal disease in Hefei city [dissertation]. Anhui: Anhui Medical University; 2009. 
49 Wang YT. Survey on carriage of Neisseria Meningitidis, Haemophilus Influenzaes, Streptococcus Pneumoniae among healthy population in Hebei province [masters dissertation]. Beijing: Chinese Center for Disease Control and Prevention; 2013.

50 Jiang X, Sun YQ, Qian ZY, Zhen SJ, Wang YT, Jia ZY, et al. Surveillance on antibody level against Meningcoccus among healthy population in three city of Hebei province. Chinese Journal of Health Laboratory Technology. 2011;21:164-66.

$51 \mathrm{Lv}$ J, Yang HM, Jiang YZ, Zou WJ, Zhao MJ, Zhu BQ, et al. Analysis of the result on surveillance of epidemic cerebrospinal Meningitis in Hubei, 2006-2010. Journal of Pathogen Biology. 2012;7:287-90.

52 Li QW, Yuan WH, Zheng EH, Yuan L, Lin ZY, Chen AP. Monitoring and analysis of epidemic cerebrospinal Meningitis infection rate and antibody level in healthy population in Fujian province in 2012. Chinese Journal of Health Laboratory Technology. 2014;24:244-6.

53 Li YC, Huang SZ, Ye XY, Zhang LP, Yang L, Fang CY. Analysis on serum bactericidal antibody level of Neisseria Meningtides serogroup C in healthy population of Dongguan city in 2010. Chinese Journal of Health Laboratory Technology. 2012;22:2957-65.

54 Li WD, Li JM, Yang KX. Analysis on surveillance of antibody level of groups A and C epidemic cerebrospinal Meningitis of healthy population in Longgang of Shenzhen. Mod Prev Med. 2010;37:2103-8.

55 Chen W, Jin LY, Qi XY. Investigation on antibody levels against epidemic cerebrospinal Meningitis and appraisal on immune efficacy among the healthy population in Tianjin. Mod Prev Med. 2010;37:3738-49.

56 Hou TJ, Wang LL. Epidemiological characteristics and disease control of meningitis in Xi'an. Chin J Microbiol Immunol. 2014;34:713-5.

57 Zhang H, Hang H, Xu QY. Epidemiological characteristics of cerebrospinal Meningitis in Suzhou. Mod Prev Med. 2006;33:1864-6.

58 Fan YC, Li C, Yan Z, Yan SH. Surveillance and analysis of epidemic cerebrospinal Meningitis in Inner Mongolia autonomous region during 1991-2010. Mod Prev Med. 2012;39:5527-31.

59 Jiang HB. Analysis of monitoring result of epidemic cerebrospinal Meningitis in Lianyungang City during 20042009. Mod Prev Med. 2012;39:1078-80.

60 Ma HS, Sun YQ, Liu HB, Li YP, Zhen SJ, Jiang X, et al. Analysis of epidemic cerebrospinal Meningitis deaths in Hebei province during 2005-2009. Mod Prev Med. 2011;38:833-9.

61 Mo SX, Sun LY, Zeng XX, Fu ZW, He J, Li J, et al. Analysis on the cerebrospinal Meningitis antibody level of health population in Hainan in 2007. Mod Prev Med. 2010;37:2913-5.

62 Yin HJ, Shi J, Gao GH, Xu XM, Chu G, Qiu J, et al. Analysis of knowledge and health situation of epidemic cerebrospinal meningitis carrier among passengers. Chin J Prev Med. 2008;42:139-41.

$63 \mathrm{Gu}$ WW. Investigation on the immune level of the Meningitis types A and C among healthy people in Shiyan city. Mod Prev Med. 2008;35:3193-6.

64 Wang XP, Jin YH, Wang H. Analysis on epidemic characteristics of epidemic cerebrospinal meningitis from 2004-2006 in Hefei. Mod Prev Med. 2008;35:2011-3.

65 Guo F, Liu XH, Jiang B, Peng B, Huang XM, Wang YG, et al. Analysis on the surveillance results of epidemic cerebrospinal meningitis in the healthy people in Suizhou city from 2001 to 2006. Chin J Dis Control Prev. 2007;11:482-4.

66 Liao GD, Liao XJ, Liu MZ, Luo XM. Investigation on the immune level of the Menitis bacterium types A and C among healthy people in Maoming, Guangdong. Chin J Epidemiol. 2006;27:180.

67 Jafri RZ, Ali A, Messonnier NE, Tevi-Benissan C, Durrheim D, Eskola J, et al. Global epidemiology of invasive meningococcal disease. Popul Health Metr. 2013;11:17. Medline:24016339 doi:10.1186/1478-7954-11-17

68 Su LY, Huang RN, Wang C, Zhang XC. [Epidemiology of Meningococcal Disease in Chengdu, 1950-2008]. [Article in Chinese]. J Prev Med Inf. 2010;26:116-20.

69 Ze W. [Planning Immunology (2nd edition)]. [Book in Chinese]. Shanghai: Shanghai Science and Technology Publishing House. 2001:465-70.

70 Yazdankhah SP, Caugant DA. Neisseria meningitidis: an overview of the carriage state. J Med Microbiol. 2004;53:821-32. Medline:15314188 doi:10.1099/jmm.0.45529-0

71 Trotter CL, Greenwood BM. Meningococcal carriage in the African meningitis belt. Lancet Infect Dis. 2007;7:797803. Medline:18045562 doi:10.1016/S1473-3099(07)70288-8

72 Espinosa de los Monteros LE, Aguilar-Ituarte F, Jimenez-Rojas LV, Kuri P, Rodriguez-Suarez RS, Gomez-Barreto D. Prevalence of Neisseria meningitidis carriers in children under five years of age and teenagers in certain populations of Mexico City. Salud Publica Mex. 2009;51:114-8. Medline:19377737 doi:10.1590/S003636342009000200006

73 Almeida-González L, Franco-Paredes C, Perez LF, Santos-Preciado JI. [Meningococcal disease caused by Neisseria meningitidis: epidemiological, clinical, and preventive perspectives]. [Article in Spanish]. Salud Publica Mex. 2004;46:438-50. Medline:15521528 doi:10.1590/S0036-36342004000500010

74 Sáfadi MA, Cintra OA. Epidemiology of meningococcal disease in Latin America: current situation and opportunities for prevention. Neurol Res. 2010;32:263-71. Medline:20406604 doi:10.1179/016164110X12644252260754

75 Díaz J, Cárcamo M, Seoane M, Pidal P, Cavada G, Puentes R, et al. Prevalence of meningococcal carriage in children and adolescents aged 10-19 years in Chile in 2013. J Infect Public Health. 2016;9:506-15. Medline:26819097 doi:10.1016/j.jiph.2015.12.011 
76 Rodriguez P, Alvarez I, Torres MT, Diaz J, Bertoglia MP, Carcamo M, et al. Meningococcal carriage prevalence in university students, 1824 years of age in Santiago, Chile. Vaccine. 2014;32:5677-80. Medline:25148776 doi:10.1016/j.vaccine.2014.08.015

77 Araya P, Diaz J, Seoane M, Fernandez J, Terrazas S, Canals A, et al. [Laboratory surveillance for invasive meningococcal disease in Chile, 2006-2012]. [Article in Spanish]. Rev Chilena Infectol. 2014;31:377-84. Medline:25327189 doi:10.4067/S0716-10182014000400001

78 Christensen H, May M, Bowen L, Hickman M, Trotter CL. Meningococcal carriage by age: a systematic review and meta-analysis. Lancet Infect Dis. 2010;10:853-61. Medline:21075057 doi:10.1016/S1473-3099(10)70251-6

79 Zhang X, Shao Z, Zhu Y, Xu L, Xu X, Mayer LW, et al. Genetic characteristics of serogroup A meningococci circulating in China, 1956-2005. Clin Microbiol Infect. 2008;14:555-61. Medline:18373693 doi:10.1111/ j.1469-0691.2008.01977.x 